\title{
Resistencia a la tracción entre una resina acrílica y un material de rebase resiliente
}

Vanderlei AD, Souza ROA, Passos SP, Nogueira Jr. L, Pavanelli CA, Balducci I. Resistencia a la tracción entre una resina acrílica y un material de rebase resiliente. Rev Estomatol Herediana. 2006; 16(2):98-102

\section{RESUMEN}

El objetivo de este estudio fue evaluar el efecto de la aplicación de diferentes adhesivos en la resistencia de unión entre una resina acrílica para base de prótesis total y un material de rebase de tejido resiliente a base de silicona (Ufi Gel P, Voco, Alemania), por medio del ensayo de tracción. Se confeccionaron 30 especimenes $(60 \mathrm{~mm}$ x $10 \mathrm{~mm})$ en resina acrílica, obtenidos a partir de un patrón metálico, los cuales fueron divididos igualmente en 3 grupos: G1 - Adhesivo Sofreliner (Tokuyama, Japón), G2 - Adhesivo Ufi Gel P (Voco, Alemania) y G3 - Adhesivo Vitacoll (VITA-Zahnfabrik, Alemania). Los especimenes de resina acrílica fueron seccionados en su región central y removidos $2,5 \mathrm{~mm}$ en extensión de cada sección y, en seguida se aplicaron el adhesivo y el material de rebase entre las dos mitades de todas las muestras. Los cuerpos de prueba (cp) fueron almacenados en agua a $37^{\circ} \mathrm{C}$ durante siete días y sometidos al ensayo mecánico para analizar la resistencia a tracción en una máquina de ensayo universal (EMIC), a una velocidad de $15 \mathrm{~mm} / \mathrm{min}$. Los datos obtenidos (Kgf) fueron sometidos al análisis de varianza $(\mathrm{p}<0,05) \mathrm{y}$ al test de $\mathrm{t}$ de Student. Las medias y los desvíos patrones obtenidos fueron: G1 $(11,54 \pm 1,64)$, G2(8,91 11,7$)$ y G3(0). Se concluyó que los valores de resistencia de unión del G1 y G2 fueron estadísticamente superiores a los de G3 $(p<0,05)$ y G1 difirió del G2 $(p<0,05)$.

Palabras clave: DENTADURA COMPLETA / RESISTENCIA A LA TRACCIÓN / ALINEADORES DENTALES / RESINAS ACRÍLICAS.

Bond strength between acrylic resin and a resilient rebase material

ABSTRACT

The objective of this study was to evaluate the effect of the application of different adhesives on the bond strength between an acrylic resin for base of denture and a soft lining material based on silicone (Ufi Gel P, Voco, Germany), by means of the tensile testing. Thirty specimens' shaped-dumbbells $(60 \mathrm{~mm} \times 10 \mathrm{~mm})$ made of acrylic resin, and obtained from a metallic standard; were divided equally in 3 groups: G1 - Adhesive Sofreliner (Tokuyama, Japan), G2 Adhesive Ufi Gel P (Voco, Germany) and G3 - Adhesive Vitacoll (VITA-Zahnfabrik, Germany) The acrylic dumbbells were divided in the middle and removed $2,5 \mathrm{~mm}$ in stretch of each section and, right away, the adhesive and the stuff soft lining were applied between the two halves of all the samples. The specimens (sp) were stored in water to $37^{\circ} \mathrm{C}$ during seven days and, then, submitted to the mechanical test for analyze the tensile strength, displayed in a universal test machine (EMIC), speed of $15 \mathrm{~mm} / \mathrm{min}$. The results obtained (Kgf) were submitted to the ANOVA $(\mathrm{p}<0,05)$ and $\mathrm{t}$ (Student) tests. The medium and the standard deviation obtained were: $\mathrm{G} 1(11,54 \pm 1,64), \mathrm{G} 2(8,91 \pm 1,7)$ and $\mathrm{G} 3(0)$. Based on the analysis of the data obtained, it was concluded that the bond strength values of $G 1$ and G2 were statistically superior over $G 3(p<0,05)$ and $\mathrm{G} 1$ differed from $\mathrm{G} 2(\mathrm{p}<0,05)$.

Key words: COMPLETE DENTURE / TENSILE STRENGTH / DENTURE LINERS / ACRYLIC RESINS.

\section{Introducción}

La prótesis total (PT) es una modalidad terapéutica antigua, pero aún largamente utilizada en individuos que han sufrido perdidas dentales totales. Ese tipo de prótesis es soportada por la mucosa que recubre el hueso del reborde alveolar, por tanto, es común en ese tipo de tratamiento que el reborde presente un aumento de la reabsorción ósea y consecuente desadaptación del aparato. Por eso, la retención y la estabilidad de una PT son considerados los factores más críticos de esta modalidad de tratamiento.

El uso de resinas resilientes para rebase directo o indirecto, también conocidas como bases suaves o soft liners, es frecuente en esas próte- sis, visto que estan indicadas clínicamente por absorber la carga masticatoria que soporta la PT, distribuyéndola de una manera uniforme por los tejidos de soporte (1-3), generando menor tensión sobre la base rígida de la PT y evitando áreas localizadas de concentración de tensión sobre el reborde alveolar. De esa forma se disminuyó la inevitable reabsorción ósea que ocurre a lo largo del tiempo promoviendo la disminución de la fricción en la fibromucosa, dando como resultado la confortabilidad funcional para los portadores de PT $(4,5)$.

Los materiales fabricados a base de silicona han sido utilizados por un largo período $(6,7)$, porque al absorber energía en el ciclo masticatorio

\section{Aleska D Vanderlei ${ }^{1}$ Rodrigo O.A. Souza ${ }^{1}$ Sheila P. Passos ${ }^{1}$ Lafayete Nogueira Jr. ${ }^{2}$ Carlos A. Pavanelli² Ivan Balducci ${ }^{3}$}

Alumno de la Maestría en Odontología Restauradora Área de Concentración : Prótesis.

Docente del Departamento de Materiales Odontológicos y Prótesis - Disciplina de Prótesis Parcial Removible.

Docente del Departamento de Odontología Social y Clínica Infantil - Disciplina de Bioestadística y Metodología Científica.

Facultad de Odontología. Universidad de San José de los Campos - UNESP, Brasil.

\section{Correspondencia}

Aleska D. Vanderle

Av. Engenheiro Francisco José Longo, 555, AP 1306 São Dimas, São José dos Campos - SP. BRASIL. CEP 12245-000

Teléfono: 055(12) 8135-2119

e-mail: aleskavanderlei@ hotmail.com

Recibido :26 de junio del 2006

Aceptado : 10 de enero del 2007 reducen la intensidad de la fuerza transmitida a la fibromucosa. Deben presentar algunas características como buena adhesión a la base de la prótesis total (4,8-12), resiliencia permanente (8), efecto amortiguador sobre la mucosa, estabilidad dimensional, promover mínima absorción de fluidos y mínima solubilidad e inhibir el crecimiento de hongos y otros microorganismos (4).

Entretanto, las PT con bases resilientes pueden presentar problemas a lo largo del tiempo, como pérdida de resiliencia o diferentes grados de ella, porosidad y pobre resistencia a la fractura (13). El aumento de la porosidad de este material podrá causar un acúmulo de biofilm dental y colonización por Candida 
albicans $(3,14)$.

Uno de los problemas más serios con las prótesis resilientes es la falta de adhesión entre el material resiliente y la base de la dentadura $(1,15)$. Estas fallas son comúnmente encontradas en la práctica clínica, pudiendo crear una potencial superficie para el crecimiento de microorganismos, biofilm y formación de cálculo (16,17). El envejecimiento en agua, el uso de primers con el material de rebase y el comportamiento de los materiales utilizados para la confección de las bases de prótesis total son analizados en diversas investigaciones con el fin de evaluar las fallas de adhesión entre estos materiales, así como las propiedades biomecánicas y la adsorción de proteínas y microorganismos (12).

Varios estudios han investigado los factores que pueden afectar la unión entre la resina acrílica de la base de la prótesis total y el material de rebase del tejido, incluyendo la naturaleza y dirección de las fuerzas, el espesor $(18,19)$ y la resistencia al rasgamiento del material de rebase (20), la naturaleza del agente adhesivo (21) y las variaciones en la estructura de resina acrílica de la base de la dentadura $(8,18,22)$.

Es innegable que la adhesión es insatisfactoria es uno de los principales problemas en la aplicación clínica de las bases resilientes $(1,11,23)$.

El presente trabajo tuvo como objetivo evaluar el efecto de la aplicación de diferentes adhesivos en la resistencia a la tracción de la interfase de unión del sistema resina acrílica / material resiliente.

\section{Material y métodos}

A partir de un patrón metálico de $60 \mathrm{~mm}$ de largo y $10 \mathrm{~mm}$ de altura (Fig. 1), se confeccionaron 30 moldes con silicona de condensación de consistencia pesada y liviana (Silon2 APS, Dentsply Ind. y Co. USA), y a partir de ellos se produjeron 30 patrones de cera en el formato del original.

Estos patrones de cera fueron incluidos en muflas (DCL número 6®),
- Dentaria Campineiro Ltda.) de la siguiente forma : se vertió yeso tipo II en la mufla y los patrones en cera se insertaron en la superficie del yeso. Fueron posicionados tres patrones en cada mufla y se cubrieron con silicona de condensación de baja viscosidad y alta dureza para la confección de murallas (VIPI-SIL, VIPI-Br), siendo esta aplicación realizada con el auxilio de una jeringa para elastómero. En seguida, el yeso fue aislado con vaselina sólida y la contramufla se llenó con yeso común espatulado en la proporción 50g/100ml, siendo cerrada posteriormente. La mufla fue sometida al prensado de 1,5 ton durante dos horas y después del fraguado del yeso, fue abierta y los patrones de cera retirados, quedando estos impresos en la superficie del yeso y de la silicona.

La resina acrílica (Clássico, Brasil) fue manipulada según las instrucciones del fabricante (1 líquido: 3 polvo), colocada en el molde de yeso en la fase plástica y prensada de manera lenta y gradual en una prensa hidráulica de mesa hasta alcanzar 1,25 t/pol ${ }_{2}$, aguardándose una hora.

Pasado este tiempo, las muflas se transfirieron a una prensa de resortes individuales y se sometieron a un ciclo de polimerización de $72^{\circ} \mathrm{C}$ por 12 horas en una termopolimerizadora.

Después del enfriamiento de las muflas a temperatura ambiente, los bastones de resina fueron removidos, realizando el acabado con fresas y lijas, con el fin de remover los excesos de resina.

Los cortes se hicieron con discos diamantados de acero (diámetro de 22mm, espesor de 0,15mm, ref 7016, KG Sorensen, São Paulo, Brasil) con baja rotación, adecuadamente refrige-

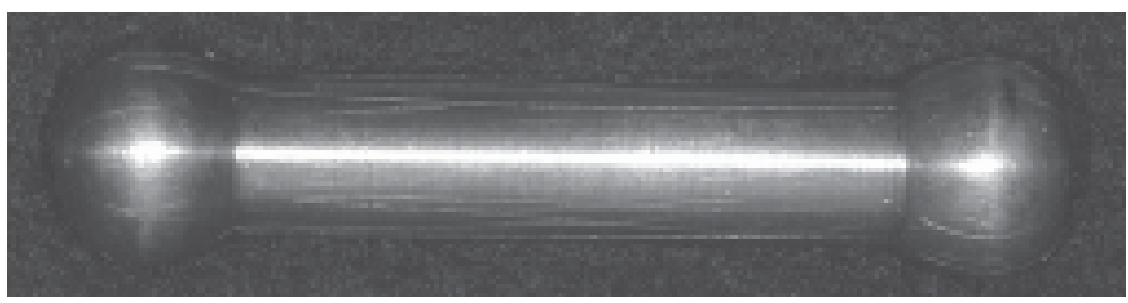

Fig. 1. Patrón metálico utilizado para confeccionar los especimenes de resina acrílica. rados con agua, colocados en una pieza de mano (Kavo Ind. e Com.Ltda) y acoplados a un torno mecánico modificado, con calibración en los ejes $\mathrm{x}$ e y, posibilitando el corte en ambas direcciones (24).

Inicialmente los cuerpos de resina acrílica fueron fijados con adhesivo de cianoacrilato (Super Bonder, Loctite ${ }^{\circledR}$, Brasil) en una base metálica cilíndrica, que a la vez fue acoplada a la garra de la máquina de corte. Fue requisito fundamental que cada patrón de resina acrílica estuviera posicionado perpendicularmente al disco diamantado, de forma con el fin de obtener los cortes más regulares posible. Después de la calibración de la máquina de corte, operación repetida en cada nuevo procedimiento de corte, se realizaron dos cortes, de manera que cada uno estuviese situado a $2,5 \mathrm{~mm}$ del centro del patrón en resina, removiendo al final una porción de $5 \mathrm{~mm}$.

Tratamiento de la superficie de la resina acrílica

Inicialmente, todas las superficies de resina acrílica que iban a entrar en contacto con el material de rebase fueron limpiadas con alcohol al 99,7\% (Voco, Cuxhaven, Germany. Lot 421394) durante 10 segundos y se esperó dos minutos antes de aplicar el adhesivo.

Los patrones de resina acrílica fueron divididos aleatoriamente en tres grupos $(\mathrm{n}=10)$, siendo cada uno sometido a la acción de un adhesivo específico: G1 (control)- adhesivo Ufi Gel P (Voco, Cuxhaven, Alemania, Lote 350291), G2 - adhesivo Sofreliner Primer (Tokuyana, Tokio, Japón, Lote 421394) y G3- adhesivo Vitacoll 
(VITA-Zahnfabrik, Alemania, Lote 987042.) Las hemisecciones de cada uno fueron adaptadas en el interior de los moldes de silicona utilizados para la confección de los patrones de cera y en el espacio entre ellas ( $5 \mathrm{~mm})$, se aplicó el acondicionador de tejidos Ufi Gel P, según recomendaciones del fabricante. Después de su polimerización los cuerpos de prueba (cp) fueron almacenados en agua destilada durante siete días, y sometidos al ensayo mecánico de tracción.

\section{Ensayo mecánico}

El ensayo fue realizado en una máquina de ensayo universal (EMIC) con capacidad de 500 kg, a una velocidad constante de $15 \mathrm{~mm} /$ min y célula de carga de $50 \mathrm{Kg}$. Además, se utilizó un dispositivo específico que ejercía la tracción en-

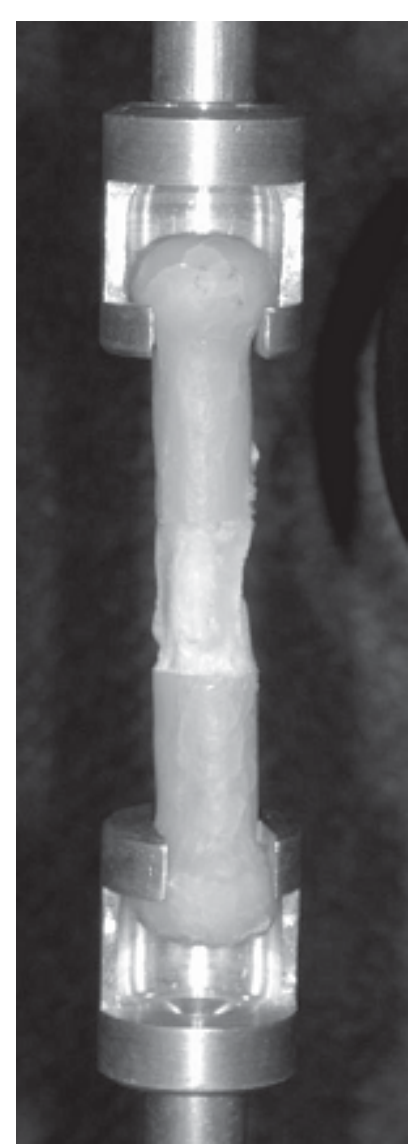

Fig. 2. Dispositivo específico que posee dos garras para sujetar los patrones de resina individualmente, sin permitir que ellos se muevan. tre dos garras que sujetaban los patrones de resina individualmente, sin permitir que se muevan (Fig. 2).

El área de todos los cp fue medida antes de la ejecución del ensayo, empleando un paquímetro digital (Starret Indústria y Comércio Ltda, Itu-SP) con precisión de centésimos de milímetro. El valor del área con el valor de carga para el rompimento de la unión fue necesario para calcular la resistencia de unión por medio de la tracción (MPa), que fue calculada por la siguiente fórmula: $\mathrm{R}_{\mathrm{t}}=\mathrm{F}$ / $A: R_{t}$ es la resistencia a la tracción; $F$, la fuerza aplicada; y A, área de unión resina/material resiliente/resina. Fue obtenida una media de resistencia a la tracción para cada conjunto y en el final fue calculada la media general para cada grupo de estudio.

Los datos obtenidos (Mpa) fueron entonces sometidos al análisis estadístico utilizándose el t de Student.

\section{Resultados}

Las medias y desviación estándar de los datos de resistencia a la tracción (Kgf) del material de rebase frente a los adhesivos utilizados, están presentados en la Tabla 1 y representados por medio del gráfico de puntos (Fig. 3), presentando $\mathrm{p}=0,003$.

Cuando se compara la distribución de los valores obtenidos en cada grupo, por medio de la prueba t de Student de muestras independientes, se verifica que los valores medios difieren estadísticamente, de manera que el G2 $(11,54 \pm 1,64)$ presentó valores de resistencia a unión significativamente mayor que los encontrados en el G1 $(8,91 \pm 1,70)$ (Tabla 1).

Por medio de la figura de arriba,

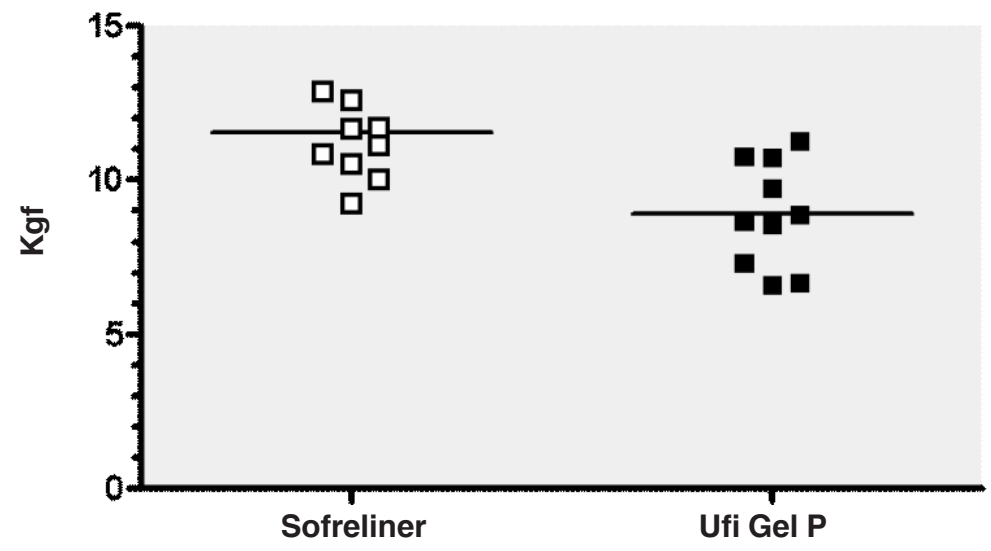

Fig. 3. Diagrama de dispersión de la columna (dot plot) de los valores de resistencia adhesiva (kgf) obtenidos en 10 cps, alrededor de la respectiva media, según el tipo de adhesivo.

Tabla 1. Datos del ensayo de tracción y los valores de las medias de resistencia a la tracción (kgf) en cada uno de los sistemas adhesivos $(\mathrm{n}=10)$.

\begin{tabular}{lcc}
\hline muestras & Sofreliner & Ufi Gel P \\
\hline 1 & 15,01 & 10,77 \\
2 & 11,64 & 8,54 \\
3 & 12,57 & 6,67 \\
4 & 11,11 & 8,86 \\
5 & 10,52 & 11,26 \\
6 & 12,87 & 6,60 \\
7 & 10,00 & 7,29 \\
8 & 11,67 & 8,66 \\
9 & 10,82 & 9,72 \\
10 & 9,23 & 10,72 \\
\hline Média \pm D.P. & $11,54 \pm 1,64$ & $8,91 \pm 1,70$ \\
$* \mathrm{p}<0,05$ & &
\end{tabular}


se puede observar: la ausencia de valores atípicos; los valores siguen una distribución simétrica alrededor del valor medio y la dispersión es próxima en todas las condiciones experimentales.

En el grupo 3 no fue posible la confección de cuerpos de prueba, porque existió unión del adhesivo Vitacoll al material de rebase. Portanto las muestras presentaron valores nulos de adhesión.

\section{Discusión}

La unión del material de rebase resiliente a la base de prótesis es un factor imprescindible para el éxito en esa modalidad de tratamiento, pero las fallas en esa interfase pueden llevar al dislocamiento del material de rebase, así como proporcionar la proliferación de bacterias, comprometiendo el resultado final.

Varios estudios demostraron que la ruptura en la adhesión entre el material resiliente y la base de prótesis, es uno de los problemas más comunes ocurridos en las prótesis rebasadas con esos materiales en la clínica diaria $(23,25,26)$.

Diversos factores influencian esta adhesión, entre ellos está el contacto con el agua, el uso de un adhesivo en la interfase resina/material de rebase y la naturaleza del material de la base de la prótesis. Según Kulak-Ozcan et al. (2003) (12), cuando son inmersos en agua pasan por dos procesos: la eliminación de monómero residual y otros elementos solubles en agua, así como la absorción de agua y saliva cuando están en el medio bucal. El equilibrio entre estos dos procesos interfiere tanto en la efectividad como en la estabilidad del sistema material/ base de la prótesis. De esa forma, los cuerpos de prueba fueron almacenados en agua destilada a 37oC durante siete días, con el propósito de mejorar la estabilidad del conjunto y simular una condición clínica.

Diversos estudios $(2,3,26)$ han evaluado la influencia de diferentes tratamientos de superficie de la resina acrílica, bien como el empleo de diferentes materiales de rebase, con el fin de mejorar la resistencia de unión entre esos materiales. Entretanto, el estudio del efecto de diferentes agentes adhesivos en la resistencia adhesiva entre un condicionador de tejidos y la resina acrílica aún es un asunto poco explorado en la literatura. En un estudio reciente realizado por McCabe et al. (2002) (11), los autores evaluaron la adhesión entre acondicionadores de tejidos a base de polivinilsiloxano (GC Reline soft, GC Reline ultrasofte GC Reline Extrasoft) frente a la utilización de diferentes agentes adhesivos y concluyeron que las muestras sometidas al tratamiento con adhesivos a base de acetato de etil presentaron mayor resistencia adhesiva cuando fueron sometidas a la misma tracción que los adhesivos a base de tolueno. Demostrando así la influencia directa del agente adhesivo en la resistencia de unión entre el acondicionador de tejidos y la resina acrílica.

En el presente estudio, a pesar de que el fabricante del acondicionador de tejidos Ufi Gel P indica el adhesivo del mismo sistema para el tratamiento de superficie de la resina acrílica, la mejor fuerza adhesiva fue encontrada en las muestras del grupo $2(11,54 \pm 1,64)$, en cuya interfase fue aplicada el adhesivo Sofreliner Primer, los cuales fueron estadísticamente superiores a los valores encontrados en el grupo 1 $(8,91 \pm 1,70)$. Por otro lado, el adhesivo Vitacoll, aplicado en las muestras del grupo 3, no promovió una unión efectiva entre el material de rebase y la resina acrílica, presentando valores nulos de adhesión, siendo todos los modos de falla del tipo adhesiva, caracterizando así, una incompatibilidad entre ese agente adhesivo y el rebasador.

Entretanto, según Sanchez (1999) (27), el valor mínimo de resistencia de unión clínicamente aceptable entre el material de rebase y la resina acrílica, es de 4,5 Kgf/ $\mathrm{cm}^{2}$ o $0,44 \mathrm{Mpa}$ ). De esa forma, a pesar de que los valores obtenidos en el grupo 2 han sido significativamente menores que los presentados en el grupo 1, ambos presentaron medias dentro de los patrones clínicos aceptables. Por otro lado, frente a las limitaciones de este estudio, se hace necesaria la realización de más investigaciones para evaluar la efectividad de los adhesivos estudiados.

\section{Conclusiones}

- El agente adhesivo Ufi Gel P presentó resistencia adhesiva significativamente menor que el adhesivo Sofreliner, pero ambos valores estuvieron dentro de los patrones clínicos aceptables.

- El agente adhesivo Vitacoll no presentó compatibilidad con el material de rebase Ufi Gel P.

\section{Agradecimientos}

Agradecemos al profesor Ivan Balducci por la realización del análisis estadístico de este estudio.

\section{Referencias bibliográficas}

1. Sinobad D, Murphy WM, Huggett R, Brooks S. Bond strength and rupture properties of some soft denture liners. J Oral Rehabil. 1992; 19(2):151-60.

2. Aydin AK, Terzioglu H, Akinay AE, Ulubayram K, Hasirci N. Bond strength and failure analysis of lining materials to denture resin. Dent Mater. 1999; 15(3):211-8. 
3. Garcia RM, Leon BT, Oliveira VB, Del Bel Cury AA. Effect of a denture cleanser on weight, surface roughness, and tensile bond strength of two resilient denture liners. J Prosthet Dent. 2003; 89(5):489-94.

4. von Fraunhofer JA, Sichina WJ. Characterization of the physical properties of resilient denture liners. Int J Prosthodont. 1994; 7(2):120-8.

5. Jagger DC, Harrison A. Complete dentures--the soft option. An update for general dental practice. Br Dent J. 1997; 182(8):313-7.

6. Murata H, Taguchi N, Hamada $\mathrm{T}$, McCabe JF. Dynamic viscoelastic properties and the age changes of long-term soft denture liners. Biomaterials. 2000; 21(14):1421-7.

7. Jepson NJ, McCabe JF, Storer $\mathrm{R}$. Age changes in the viscoelasticity of permanent soft lining materials. J Dent. 1993; 21(3): 171-8.

8. al-Athel MS, Jagger RG. Effect of test method on the bond strength of a silicone resilient denture lining material. J Prosthet Dent. 1996; 76(5):535-40.

9. Hekimoglu C, Anil N. The effect of accelerated ageing on the mechanical properties of soft denture lining materials. J Oral Rehabil. 1999; 26(9):745-8.

10. Takahashi Y, Chai J. Shear bond strength of denture reline polymers to denture base polymers. Int J Prosthodont. 2001; 14(3):271-5.

11. McCabe JF, Carrick TE, Kamohara H. Adhesive bond strength and compliance for denture soft lining materials.
Biomaterials. 2002; 23(5):1347-52.

12.Kulak-Ozkan Y, Sertgoz A, Gedik H. Effect of thermocycling on tensile bond strength of six silicone-based, resilient denture liners. J Prosthet Dent. 2003; 89(3):303-10.

13. Kawano F, Dootz ER, Koran A 3rd, Craig RG. Comparison of bond strength of six soft denture liners to denture base resin. J Prosthet Dent. 1992; 68(2):368-71.

14.Zissis AJ, Polyzois GL, Yannikakis SA, Harrison A. Roughness of denture materials: a comparative study. Int $\mathrm{J}$ Prosthodont. 2000; 13(2):136-40.

15. Kutay O, Bilgin T, Sakar O, Beyli $\mathrm{M}$. Tensile bond strength of a soft lining with acrylic denture base resins. Eur J Prosthodont Restor Dent. 1994; 2(3):123-6.

16. Taguchi N, Murata H, Hamada T, Hong G. Effect of viscoelastic properties of resilient denture liners on pressures under dentures. J Oral Rehabil. 2001; 28(11):1003-8.

17. Sertgoz A, Kulak Y, Gedik H, Taskonak B. The effect of thermocycling on peel strength of six soft lining materials. J Oral Rehabil. 2002; 29(6):583-7.

18. Kutay O. Comparison of tensile and peel bond strengths of resilient liners. J Prosthet Dent. 1994; 71(5):525-31.

19. Al-Athel MS, Jagger RG, Jerolimov V. Bone strength of resilient lining materials to various denture base resins. Int $\mathrm{J}$ Prosthodont. 1996; 9(2):167-70.

20.Baysan A, Parker S, Wright PS. Adhesion and tear energy of a long-term soft lining material activated by rapid microwave energy. J Prosthet Dent. 1998;
79(2):182-7.

21.Wood WE, Johnson DL, Duncanson MG. Variables affecting silicone-polymethyl methacrylate interfacial bond strengths. J Prosthodont. 1993; 2(1):13-8.

22. Kawano F, Dootz ER, Koran A 3rd, Craig RG. Bond strength of six soft denture liners processed against polymerized and unpolymerized poly(methyl methacrylate). Int J Prosthodont. 1997; 10(2):178-82.

23. Jagger RG, al-Athel MS, Jagger DC, Vowles RW. Some variables influencing the bond strength between PMMA and a silicone denture lining material. Int $\mathrm{J}$ Prosthodont. 2002; 15(1):55-8.

24. Andreatta Filho OD, Galhano G, Bottino MA, Camargo FP, Valandro LF, Nishioka RS. Effect of thermocycling on bond strength of an aluminous ceramic to a resin cement. Cienc Odontol Bras. 2003; 6(3):32-9.

25.Wright PS. The success and failure of denture soft-lining materials in clinical use. J Dent. 1984; 12(4):319-27.

26. Polyzois GL. Adhesion properties of resilient lining materials bonded to light-cured denture resins. J Prosthet Dent. 1992; 68(5):854-8.

27. Sanchez JLL. Estudo "in vitro" da resistência da união entre resina acrílica e materiais reembasadores resilientes submetidos ou não a termociclagem. 1999. 143 p. Dissertação (Mestrado em Prótese) - Faculdade de Odontologia de Piracicaba, Universidade Estadual de Campinas, Piracicaba. 